\title{
Fleckfieber und Fleckfieberforschung zur Zeit des Ersten Weltkrieges
}

Zum Gedenken an Henrique da Rocha Lima (1879-1956)

Von Werner Sackmann

Die ätiologische Erforschung des Fleckfiebers reicht geschichtlich nicht so weit zurück wie diejenige anderer Infektionskrankheiten. Sie fällt eindeutig in das 20. Jahrhundert und hat durch die besonderen Umstände und Bedingungen des Ersten Weltkrieges entscheidenden Auftrieb erfahren. Die ersten grundlegenden Erkenntnisse verdanken wir Amerikanern, Franzosen, Österreichern und Deutschen. Unter den in Deutschland tätigen Forschern nahm jedoch ein Ausländer eine hervorragende Stellung ein; es war der Brasilianer Henrique da Rocha Lima (sprich: «Roscha»), dessen Geburtstag sich 1979 zum hundertsten Male jährt. Sein Leben und Werk ist schon öfters gewürdigt worden. Wenn es im folgenden nochmals geschieht, so sei gleichzeitig auch vieler anderer persönlicher Schicksale gedacht, die in jener Zeit vom Fleckfieber geprägt wurden.

Da Rocha Lima wurde als Sohn eines angesehenen Arztes am 24. November 1879 in Rio de Janeiro geboren und durchlief dort 1889-1891 das Colegio Americano, dann 1892-1895 das Ginasio de Rio de Janeiro. Weil es an der brasilianischen Universität damals noch keine naturwissenschaftliche Fakultät gab, begnügte er sich mit dem Medizinstudium und schloß es 1900 mit der Doktorpromotion ab.

Nach kurzer Assistentenzeit am Hospital Misericordia trat da Rocha Lima in das Instituto Seroterapico Federal in Manguinhos ein. Die Gründung dieses staatlichen Serum- und Impfstoffwerkes war auf die Schockwirkung hin erfolgt, die ein Pestausbruch in der Stadt Santos (1899) ausgelöst hatte ${ }^{1,2}$. Erster Direktor der Anstalt war Oswaldo Goncalves Cruz (1872-1917).

Bald jedoch verließ da Rocha Lima seine Heimat und ging zur Weiterbildung nach Europa. In Berlin arbeitete er beim Pathologen Johannes Orth (1847-1923), dem Nachfolger (1902) von Rudolf Virchow und Entdecker des Kernikterus, und beim Pathologen Karl Kaiserling (1869-1942), der erstmals die lipoide Zelldegeneration beschrieb und eine farbechte Konservierung anatomischer Präparate erfand. Am Berliner Hygiene-Institut schloß da Rocha Lima sich dem Bakteriologen Philipp Martin Ficker (1868-1950) an.

Als da Rocha Lima 1903 nach Brasilien zurückkehrte, war Oswaldo Cruz gerade zum Direktor des staatlichen Gesundheitswesens ernannt worden. In dieser Eigenschaft trieb er den Ausbau des Forschungsinstitutes in Manguinhos voran, 
das später nach ihm benannt werden und Weltruf erlangen sollte ${ }^{3,4}$. Die Hauptaufgabe der Anstalt war zunächst die Bekämpfung des Gelbfiebers und die Herstellung verschiedener Heilseren und Impfstoffe; weiterhin hatte sie der Spezialausbildung von Ärzten in der Diagnostik und Bekämpfung von Seuchen zu dienen. Da Rocha Lima wurde als Abteilungsdirektor verpflichtet und hatte als Kursleiter Anteil am Unterricht.

Im Jahre 1909 begab er sich abermals nach Deutschland. Diesmal wurde er Schüler des Münchener Pathologen Hermann Ludwig Friedrich Duerck (1869-1941). Wie verschiedene deutsche Gelehrte, z. B. der Pharmazeut Gustav Giemsa (18671948) und der Zoologe Max Hartmann (1876-1962), so war auch Duerck vormals am Instituto Oswaldo Cruz Gastdozent gewesen. Da Rocha Lima begleitete Duerck auch nach Jena, als dieser dort einen Lehrstuhl an der Universität übernahm. Bald danach trat er jedoch an das Hamburger Tropeninstitut über. Eingeladen hatte ihn Stanislaus (nicht Ladislaus; vgl. ${ }^{5}$ ) Josef Matthias Prowazek, Edler von Lanow (1875-1915) ${ }^{6-13}$, den er ebenfalls in Brasilien kennengelernt hatte.

Wie da Rocha Lima hatte auch der damals 35jährige Protozoologe von Prowazek eine vorzügliche Ausbildung hinter sich. In dem Jahrzehnt seit seiner Doktorpromotion (1899) bei Berthold Hatschek (1854-1941) in Wien hatte er bei Paul Ehrlich in Frankfurt a.M., bei Richard Hertwig in München und bei Fritz Schaudinn in der Meeresstation Rovigno (Istrien), am Reichsgesundheitsamt in Berlin und am Hamburger Tropeninstitut gearbeitet. Auch hatte er 1907 an der zweiten Luesexpedition des Breslauer Dermatologen Albert Neisser (18551916) nach Java teilgenommen und dort gemeinsam mit Ludwig Halberstaedter (1877-1949), einem Assistenten Neissers, den Erreger des Trachoms entdeckt ${ }^{14,15}$.

Mit dem Fleckfieberparasiten, den er zusammen mit dem Trachomerreger und anderen Viren zu den sog. Chlamydozoen einreihte ${ }^{16,17}$, beschäftigte er sich 1913 in Serbien und angeblich auch in Sibirien ${ }^{18}$, falls sich hier nicht eine Verwechslung von Ländernamen eingeschlichen hat. Wäre v. Prowazek ein längeres Leben beschieden gewesen, so hätte er seine Beobachtungen zur Ätiologie des Flecktyphus gewiß ausführlicher niedergeschrieben und würde heute womöglich als Entdecker gelten, denn zweifellos hat er schon die Feststellungen gemacht, die kurz nach seinem Tode da Rocha Lima berichtete.

Das 1900 von Bernhard Nocht (1857-1945) gegründete Institut für Schiffsund Tropenkrankheiten in Hamburg ${ }^{19}$ wurde da Rocha Limas Wirkungsstätte für volle 18 Jahre. Von 1914 an leitete er dort eine eigene Abteilung für Pathologie; an der neugegründeten Hamburger Universität habilitierte er sich 1919 als Privatdozent. Vordem war ihm schon der Professortitel ehrenhalber verliehen worden, eine Ehrung, die hin und wieder vorkam. Munk ${ }^{20}$ nennt zunächst als einzigen 
Parallelfall denjenigen von Shibasaburo Kitasato (1852-1931) am Robert KochInstitut (um 1890), erwähnt dann aber an anderer Stelle auch die Titularprofessur des jungen Paul Ehrlich an der Charité (1884) ${ }^{11,20}$; nachzutragen wäre auch diejenige des Chirurgen James Israel (1848-1926) am jüdischen Krankenhaus in Berlin (1894) ${ }^{21}$.

Am Anfang seiner Wirksamkeit galt da Rocha Limas Interesse vornehmlich dem Gelbfieber und seiner pathologisch-anatomischen Diagnose. Er beschrieb typische, herdförmig disseminierte Nekrosen im Leberparenchym ${ }^{22-25}$, die zuweilen auch nach ihm benannt wurden ${ }^{26-28}$. Nebstdem befaßte er sich mit der Bartonellose $^{29-32}$, den Trypanosoma-Infektionen ${ }^{33-35}$, den tropischen Mykosen ${ }^{36}$ und mit parasitären und infektiösen Dermatosen ${ }^{37,38}$. Das von Samuel Taylor Darling (1872-1925) in Panama entdeckte und zunächst als Protozoon (Leishmania) angesprochene Histoplasma capsulatum ${ }^{39}$ hat er beweiskräftig den Pilzen zugeordnet; auch wies er auf die Verwandtschaft zum Erreger der Lymphangiitis epizootica der Pferde hin ${ }^{40,41}$. Eine seiner letzten Arbeiten ist die imposante Übersicht über die «Methoden der Virusforschung», die er gemeinsam mit José Reis und Karl Silberschmidt zu Emil Abderhaldens Handbuch der biologischen Arbeitsmethoden beigesteuert hat ${ }^{42}$.

In der Mehrzahl seiner wissenschaftlichen Arbeiten beschäftigt sich da Rocha Lima jedoch mit dem Fleckfieber, mit dessen $\ddot{A t i o l o g i e}^{43,44}$ und Epidemiologie ${ }^{45,46}$. Obgleich die klinische Kenntnis dieser Seuche bis in die Antike zurückreicht ${ }^{47}$, ist ihre wissenschaftliche Erforschung und Aufklärung doch verhältnismäßig neueren Datums. Sie beginnt etwa mit Howard Taylor Ricketts (1871-1910)11,48-51, Professor für Pathologie an der Universität Chicago, und seinem jungen Mitarbeiter Russell Morse Wilder (1885-1959) ${ }^{52,53}$, sowie mit dem Direktor des Pasteur-Instituts in Tunis und späteren (1928) Nobelpreisträger Charles H.J. Nicolle (1866-1936) ${ }^{54}$ und dessen beiden Kollegen Ernest Conseil (1879-1930) ${ }^{55}$ und Alfred Conor (1870-1914) ${ }^{56}$. Mit der Epidemiologie des Rocky Mountain Spotted Fever ${ }^{57,58}$, der Fièvre boutonneuse ${ }^{59,60}$ und ähnlicher übertragbarer Krankheiten vergleichend, stellten diese Forscher fest, daß der Erreger des Flecktyphus durch Läuse von Mensch zu Mensch verbreitet wird ${ }^{61}$ und daß die Krankheit auch künstlich mit Fieberblut bzw. Läusekot auf Affen ${ }^{62}$ und Meerschweinchen ${ }^{63}$ übertragen werden kann. Daß Flecktyphus durch Überimpfung von Patientenblut auf gesunde Menschen übertragbar ist, wußte man schon seit den Selbstversuchen, die der russische Arzt J.Motschutkowski (1845-1903), später Professor für Neurologie in St.Petersburg, im Jahre 1876 ausgeführt hatte ${ }^{64}$. Wie durch Meerschweinchenversuche bewiesen wurde, ist Patientenblut schon gegen Ende der 10 bis 14 Tage dauernden Inkubationszeit infektiös ${ }^{65}$. 
Im 19. und im beginnenden 20. Jahrhundert war Europa namentlich von Osten und Südosten her vom Flecktyphus bedroht. Als Begleiterscheinung und Folge so vieler Kriege, ja als entscheidender Faktor für ihren Ausgang ${ }^{66,67}$, flammte er auch in den beiden Balkankriegen, 1912 und 1913, wieder auf. In diesen bewaffneten Konflikten verlor das Osmanische Reich seine Provinzen Albanien und Mazedonien an das bulgarische Zarenreich. Im Verein mit den übrigen Balkanstaaten, abgefallenen Bundesgenossen der Bulgaren, machten die Türken sodann den erfolglosen Versuch, ihre verlustreiche Niederlage wieder wettzumachen.

Auf den Kriegsschauplätzen des Balkans sammelte von Prowazek seine ersten Erfahrungen über den äußeren und inneren Krankheitsverlauf beim Flecktyphus ${ }^{68,69}$. Er wurde dabei unterstützt durch den Internisten Carl Theodor Hegler (1878-1943) ${ }^{70,71}$. Hegler war damals am Eppendorfer Krankenhaus tätig. Er wurde später Chefarzt am Krankenhaus St. Georg in Hamburg, wo er gemeinsam mit Gerhard Domagk die klinische Erprobung der ersten Sulfonamidpräparate vornahm ${ }^{72,73}$.

Im Sommer 1914 entsandte das Hamburger Tropeninstitut zusammen mit von Prowazek auch da Rocha Lima als Hygieneexperten und Berater der Generäle und Ärzte des osmanischen Heeres nach Konstantinopel. Die Streitkräfte waren durch die Balkankriege arg mitgenommen und überdies schon von früheren Kriegszügen her stets mit Flecktyphus durchseucht ${ }^{74}$. Im Interesse zunehmender Annäherung sollte das Heer des von den Mittelmächten umworbenen Reiches am Bosporus, das im November 1914 in den Krieg eintrat, raschestens erstarken. Nach dem Fanal von Sarajevo eilten die beiden Experten jedoch wieder in ihre Wahlheimat zurück, wahrscheinlich um die zu den Fahnen gerufenen deutschen Kollegen am Tropeninstitut zu vertreten.

Nach Hindenburgs Sieg bei Tannenberg wurde der Flecktyphus schon im August 1914 auch für Mitteleuropa zur akuten Gefahr. Durch Massen russischer Kriegsgefangener wurde die Seuche nämlich mit einem Male mitten ins Deutsche Reich hinein verschleppt. Die Hamburger Experten waren jetzt auch im eigenen Lande gefragte Leute. So wurden von Prowazek und da Rocha Lima im ersten Kriegswinter als Hilfsärzte in das Gefangenenlager Merzdorf bei Cottbus in der Niederlausitz (ca. $100 \mathrm{~km}$ südöstlich von Berlin) entsandt, wo sie gleich vielen anderen Lazarett- und Lagerärzten bald selbst von der Ansteckung ereilt wurden. Von Prowazek starb am 17. Februar $1915^{75,86}$. Da Rocha Lima kam mit dem Leben davon und wurde hernach für seinen mutigen Einsatz im Seuchenkampf mit dem Eisernen Kreuz ausgezeichnet.

Der bedauerliche Ausgang der Episode von Cottbus wurde u. a. auch von Prof. Georg Johann Jürgens (1870-1966) ${ }^{76-78}$ beklagt ${ }^{79}$. Jürgens war Schüler von Ru- 
dolf Virchow, Max Rubner und Robert Koch gewesen und wurde 1916 Chefarzt im Berliner Krankenhaus am Urban. Er war insbesondere bekannt durch seine Lehrbücher über Infektionskrankheiten ${ }^{80}$ und Epidemiologie ${ }^{81}$ sowie durch seine Monographie über das Fleckfieber ${ }^{82}$.

Wie in Cottbus wütete das Fleckfieber in zahlreichen anderen Gefangenenlagern und -lazaretten, so in Weinberge ${ }^{83}$, in Marchtrenk bei Linz ${ }^{84}$ und in Görlitz ${ }^{85}$, ferner - nach den Verlustlisten ${ }^{86}$ zu schließen - in Schneidemühl (Posen), Gardelegen (bei Magdeburg), Langensalza (Thüringen) und Kassel; in Kassel kostete der Flecktyphus nicht weniger als vier Ärzten das Leben.

Wie andere Offiziere dürften auch Truppen- und Lagerärzte unter der Läuseplage zunächst weniger gelitten haben als die Mannschaften, Lagerinsassen, Verwundeten und Kranken; doch waren sie bei ihrem Umgang mit zahllosen Patienten besonders gefährdet ${ }^{87}$. Oft schien der vorübergehende Befall mit einer einzigen Laus zur Infektion zu genügen ${ }^{88,89}$. Mitunter waren auch aerogene Infektionen mit eingetrockneten Läusefaeces, die an der Außenwelt monatelang infektiös bleiben können ${ }^{90-92}$, eindeutig nachzuweisen.

Unter den 1724 Militärärzten z.B., die laut dem Sanitätsbericht des deutschen Heeres $^{86}$ im Kriegsdienst 1914-1918 gefallen bzw. verstorben sind, figurieren 210 Opfer seuchenhafter Krankheiten. Von diesen starben an Fleckfieber fast die Hälfte, nämlich 99. Diese Krankheit kostete somit gleichviel Todesopfer wie Grippe, Abdominaltyphus und Ruhr zusammen.

Ziemlich genau die Hälfte der vom Fleckfieber dahingerafften Ärzte hatten ihren Dienst in Lazaretten und Gefangenenlagern getan. Im Zusammenhang mit dem Lager bei Cottbus führen die Verlustlisten außer von Prowazek noch zwei weitere Ärzte an, nämlich den Ende 1914 verstorbenen Unterarzt Willy Hirschfeld und den fachärztlichen Beirat des 3.Armeekorps, Prof. Georg Jochmann (1874-1915) ${ }^{76,86,93}$. Jochmann hatte noch Hirschfelds Obduktion vorgenommen, sich aber wahrscheinlich erst bei einer letzten Lagerinspektion in Brandenburg infiziert. Aus den zwei Wochen seines Krankenlagers hat er der Nachwelt ein gewissenhaft geführtes medizinisches Tagebuch hinterlassen ${ }^{94,95}$. Als Internist am Rudolf Virchow-Krankenhaus und als Mitglied des Robert Koch-Institutes in Berlin war er einer der besten Experten für Infektionskrankheiten und Seuchenlehre. Soeben war noch sein Lehrbuch der Infektionskrankheiten erschienen ${ }^{96}$, das später von Carl Hegler neu bearbeitet wurde ${ }^{97}$. Seine bakteriologische Ausbildung hatte Jochmann bei Bernhard Fischer (1852-1915) erhalten; er entstammte also der sogenannten Kieler Schule, die sich um die Differentialdiagnostik der Typhus-Paratyphus-Enteritisbakterien bleibende Verdienste erworben hat ${ }^{98,99}$. 
Zu den jüngeren Kieler Kollegen Jochmanns zählte auch Paul Neukirch (geb. $1885)^{76,77}$, der während des Weltkrieges nicht nur die nach ihrem anatolischen Fundort Erzindjan benannten Paratyphus C-Bakterien beschrieb ${ }^{100-102}$, sondern in seinem nahöstlichen Einsatzgebiet auch die Schutzimpfung mit inaktiviertem Fleckfieberserum erprobte ${ }^{103,104}$.

Auf einer Hilfsexpedition des deutschen Roten Kreuzes nach Südrußland verstarb am 10. Dezember 1921 an Flecktyphus der 31jährige Marineoberarzt und Hygieniker Dr. Wolfgang Gärtner ${ }^{105,106}$. Auch Gärtner gehörte der Kieler Schule an. Er war der Sohn von Prof. August Gärtner in Jena, der anläßlich einer Fleischvergiftungsepidemie 1888 die Salmonella enteritidis entdeckt hatte.

Am Fleckfieber verstarb Ende 1916, kurz nach seiner Rückkehr von der Ostfront, der Reservearzt Arthur Pappenheim, ein angesehener Hämatologe, der mit seinen 46 Jahren außer der nach ihm benannten panoptischen Färbemethode für Blutausstriche (May-Grünwald-Giemsa) ein Opus von über hundert wissenschaftlichen Arbeiten hinterlie ${ }^{86,107}$. Als Opfer des Flecktyphus wird in der Fachliteratur auch der vertretende Militärarzt Georg Cornet (1858-1915) beklagt. Cornet war seit den achtziger Jahren Mitglied des Robert Koch-Institutes und namentlich bekannt durch seinen Nachweis von Tuberkelbazillen an der Außenwelt $^{76,86,93,94}$.

Während die bisher genannten Opfer des Flecktyphus in den engeren Fachkreisen betrauert wurden, hat der Tod der nachgenannten Persönlichkeit Aufsehen und Bestürzung in der breiten Öffentlichkeit hervorgerufen. Es betraf dies den preußischen Generalfeldmarschall Colmar Freiherr von der Goltz (18431916), den langjährigen militärischen Berater in der Türkei (1883-1895). Im Weltkrieg war er Chef einer deutschen Militärmission bei der Hohen Pforte und Oberbefehlshaber der osmanischen Streitkräfte. Er war es, der diese siegreich nach Mesopotamien führte und die Engländer bei Kut-el-Amara vernichtend schlug. Die Kapitulation des Feindes, Ende April 1916, erlebte er indessen nicht mehr, denn in Bagdad erlag der 73jährige Feldherr in der Nacht vom 18. zum 19. April dem Flecktyphus, den er sich während der mühsamen Rückreise auf dem Tigris zugezogen hatte ${ }^{93,108}$.

Daß Flecktyphus durch Läuse, in erster Linie durch die Kleiderlaus, aber auch durch die Kopflaus ${ }^{109}$ übertragen wird und nicht durch Zecken wie das Rocky Mountain Spotted Fever, hatten schon Ricketts und Wilder ${ }^{110,111}$ sowie Nicolle und seine Mitarbeiter erkannt ${ }^{112^{-114}}$. Im Mikroskopausstrich des Patientenblutes einen Erreger zu erspähen, gelang ihnen jedoch niemals eindeutig. Hingegen gelang die Darstellung von mikroskopisch knapp sichtbaren, nach Giemsa färbbaren und Bakterienfilter nicht passierenden Stäbchen bzw. Diplokokken im 
Magendarmepithel der Laus, wo sie sich massiv vermehren und anreichern, bis die Wirtszellen platzen und die Mikroben mit den Exkrementen ausgeschieden werden. Diesen Vorgang hat da Rocha Lima in allen Einzelheiten geklärt und epidemiologisch richtig gedeutet ${ }^{45,115,116}$. An seinen Laboratoriumsarbeiten hatte eine junge Mitarbeiterin wesentlichen Anteil, nämlich die Zeichnerin Hilda Sikora (1889-1978) $)^{46,117-119}$.

Aufgrund ihrer intrazellulären Vermehrung konnte da Rocha Lima die festgestellten Mikroorganismen mit dem Flecktyphus in ursächlichen Zusammenhang bringen und sie von den extrazellulären Organismen (Rickettsia pediculi) unterscheiden, die Ricketts und Wilder auch in gesunden, nicht infektiösen Läusen gesehen hatten. Mikroben, die nur in Flecktyphusläusen auffindbar waren, hatten übrigens schon 1914 Edmond Sergent (geb. 1876), Henri Foley (1871-1956) ${ }^{120}$ und C. Vialatte beobachtet ${ }^{121}$.

Der eigentliche Infektionsakt wurde vielfach im blutsaugenden Stich der Laus vermutet. Da die Fleckfieber-Parasiten jedoch aus dem Darm austreten, ist der Stich nur insofern gefährlich, als rickettsienhaltige Faeces und Inhalt von zerdrückten Läusen in denselben eingerieben werden. Die Erreger können ebensogut andere Verletzungen als Eintrittspforte benützen, wie dies auch der Erreger des Läuserückfallfiebers, Borrelia recurrentis, tut. Bezüglich des letzteren war anscheinend auch da Rocha Lima noch der irrigen Meinung, die Spirochäten träten unmittelbar aus der Speicheldrüse der Laus in den menschlichen Blutkreislauf über $^{45}$; doch trifft dies nur für die durch Zecken übertragene Borrelia duttoni $\mathrm{zu}^{122}$.

Den Mikroorganismus, den da Rocha Lima erstmals als spezifischen Erreger des Fleckfiebers ansprach, belegte er zur Erinnerung an seinen verstorbenen Kollegen von Prowazek und zu Ehren von H.T. Ricketts, der sich ebenfalls eine tödliche Flecktyphusinfektion zugezogen hatte, mit dem wissenschaftlichen Namen Rickettsia prowazekii ${ }^{43,123}$. Bezeichnenderweise wurde demnach eine Benennung gewählt, die wohlweislich keinerlei Bezug zur Erregerrolle des Parasiten und zur Krankheit nimmt. Diese Beziehungen waren erst noch stichhaltiger zu beweisen $^{124,125}$.

Den besonderen, völlig neuartigen Gattungsnamen Rickettsia wählte da Rocha Lima wohl in der Voraussicht, daß es sich hier nicht um einen gewöhnlichen Bazillus oder Diplokokkus handelte ${ }^{116,126}$. Er hielt es zwar noch nicht für endgültig ausgeschlossen, daß die Rickettsien, die seither zwar stets als besondere Mikrobengruppe aufgefaßt wurden, Bakterien seien ${ }^{44}$; doch hat er sie diesen nie eindeutig zugezählt ${ }^{127,128}$. Gerade das wurde ihm aber sehr verübelt, so z. B. von Hans Willi Töpfer und Hermann Schüßler, welch letzterer sich noch 1916 als 
Assistent am Kaiser Wilhelm-Institut für Biologie eine tödliche Laborinfektion mit Flecktyphus zuzog ${ }^{129,130}$. Mit diesen beiden Kollegen hatte da Rocha Lima einen erbitterten Prioritätsstreit bezüglich der Entdeckung von Rickettsia prowazekii auszufechten ${ }^{131,132}$.

Die Frage nach der Bakteriennatur des Flecktyphuserregers war deshalb von besonderer Aktualität, weil der eindeutigen ätiologischen Klärung zahlreiche Meldungen vorausgingen, die verschiedenste zufällig vorgefundene Bakterien zu Erregern erklärten ${ }^{116,133}$. Klinisch wurde der Flecktyphus auch häufig mit dem Abdominaltyphus verwechselt. Anderseits erwiesen sich zahlreiche dem Fleckfieber zugeschriebene Fälle nach der bakteriologischen Untersuchung als Typhusbzw. Paratyphuserkrankungen ${ }^{134}$. Auch andere gramnegative Stäbchenbakterien wurden häufig aus Fleckfieberpatienten isoliert.

Die Beschreibungen der aus Fleckfieberkranken gezüchteten Bakterienarten zu durchgehen, ist heute kaum mehr von Interesse. Einer dieser Keime, insbesondere aber seine Entdecker müssen jedoch erwähnt werden, nämlich der Arzt Edmund Weil (1879-1922) ${ }^{135,136}$ und der Chemiker Arthur Felix (1887-1956) ${ }^{137-}$ 139. Weil und Felix lernten sich 1915 als Mitglieder des Epidemielaboratoriums Nr. 5 der II. k. k. Armee in Galizien kennen ${ }^{134-138}$. Ende 1915 isolierten sie aus dem Urin eines Fleckfieberkranken ein unbewegliches, später als Proteus identifiziertes Bakterium, das im Serum von Fleckfieberpatienten scheinbar spezifisch agglutinierte $^{140,141}$.

Weil und Felix vermeinten zwar nur vorübergehend, damit die Ursache des Fleckfiebers entdeckt zu haben. Gegen die Erregerrolle der Proteuskeime wurden alsbald Einwände vorgebracht, nicht zuletzt aufgrund der Selbstversuche von Richard Hamburger (geb. 1884) ${ }^{77}$ und Robert Bauch (1897-1957) 77, 78, 142, 143 sowie von Max H. Kuczinski (geb. 1890) ${ }^{64,77}$; aber die von Oskar Bail ${ }^{144}$ erstmals nach Weil und Felix benannte Reaktion wurde ein verläßliches Mittel zur serologischen Diagnose der Krankheit. Felix erprobte sie in der Folge nicht nur an der Ostfront, sondern zeitweilig auch im deutschen Rotkreuzlazarett in Konstantinopel ${ }^{103,145}$. Das Phänomen beruht auf alkalistabilen Lipopolysacchariden (OAntigenen), die den Proteuskeimen und dem Flecktyphuserreger gemeinsam sind, obschon diese sonst keineswegs verwandt sind ${ }^{146-149}$. Weder Vakzinen von Proteus $\mathrm{OX}_{19}$ noch Antiseren gegen diesen Keim verleihen einen Schutz gegen die Flecktyphusinfektion, wie Doerr und Pick in Meerschweinchenversuchen feststellten $^{65}$. Wie Viktor Karl Ruß ${ }^{150}$ gemeinsam mit L. Kirschner zeigte, geben Versuchstiere auch nicht so leicht eine positive Weil-Felix-Reaktion ${ }^{151}$.

Die kriegsbedingte Aktualität der Fleckfieberseuche verhalf der Entdeckung von Weil und Felix offenbar zu solcher Publizität, daß die Mit- und Nachwelt 
den Umstand übersehen hat, daß diese Entdeckung schon einmal gemacht worden war. Bereits 1909 hatte nämlich der Engländer William James Wilson (18791954) $)^{152-154}$, nach dem ein Salmonella-Nährboden benannt ist ${ }^{154}$, heterologe Agglutinine im Serum von Fleckfieberkranken beschrieben ${ }^{155,156}$.

Es bleibt nachzutragen, daß Weil und Felix, ausgehend von dem unbegeißelten Proteus $\mathrm{X}_{19}$, die thermostabilen, somatischen Antigene der Proteus- und anderer Bakterien von den geißelständigen zu unterscheiden begannen und sie erstmals als O- bzw. H-Antigene bezeichneten ${ }^{157,158}$. Auch diese Verschiedenheit der Antigene war indessen schon bekannt; sie war von den Amerikanern Theobald Smith (1859-1934), Henry Gustav Beyer (1850-1918) und A.L. Reagh, ebenso von A. Joos in Brüssel, schon beobachtet worden ${ }^{159-161}$.

Ein weiterer großer Wurf gelang Felix 1934 am Lister-Institut in London, als er zusammen mit Margareth Pitt das Vi-Antigen von Salmonella typhi entdeckte ${ }^{162}$. Weil seinerseits kommt das Verdienst zu, den Paratyphus C-Erreger, von ihm als Bacillus paratyphosus $\beta_{5}$ bezeichnet, entdeckt zu haben ${ }^{163}$. Diese auch von Paul Neukirch in Anatolien beschriebene Species ${ }^{100,101}$ wird demnach heute ${ }^{164}$ zu Unrecht nach Ludwik Hirszfeld (1884-1954) benannt, der sie 1919 in Thrazien angetroffen und die Bezeichnung "Paratyphus C» eingeführt hat ${ }^{165}$. Weil starb, nach Prag zurückgekehrt, 1922 an einer okulären Laborinfektion mit Rickettsia prowazekii; dasselbe Schicksal ereilte 14 Jahre später im gleichen Laboratorium am Prager Hygieneinstitut seinen Schüler und Nachfolger Friedrich Breinl $(1888-1936)^{11,166,167}$.

Der ätiologische Zusammenhang zwischen dem obligaten Zellparasiten im Darm der Laus und der Flecktyphuserkrankung beim Menschen wurde schließlich endgültig bewiesen durch den Amerikaner Simeon Burt Wolbach (18801954) ${ }^{168-171}$ und durch seine Mitarbeiter Monroe Jacob Schlesinger (18921955) ${ }^{172}$, Francis Winslow Palfrey (1876-1953) ${ }^{173}$ und John Lancelot Todd (1876-1949 ${ }^{174}$. Letzterer hatte sich schon anfangs des Jahrhunderts durch seine gemeinsam mit dem Engländer Joseph Everett Dutton (1874-1905) unternommenen Expeditionen in die Trypanosomaherde Senegambiens und des Kongo einen Namen gemacht und war später Professor an der McGill-Universität in Toronto.

Den amerikanischen Forschern, die anfangs der zwanziger Jahre bei der Fleckfieberbekämpfung in Polen erfolgreich mitgewirkt hatten ${ }^{175}$, gelang die Züchtung der Rickettsien in Kulturen und Subkulturen von Gehirnendothelien infizierter Meerschweinchen. Mit solchen Kulturen, unter Umgehung der Laus also, ließ sich wiederum Flecktyphus erzeugen ${ }^{176}$. Dasselbe gelang auch - unter Umgehung der Zecke - mit dem Erreger des Rocky Mountain Spotted Fever, Rickettsia 
rickettsii, in endothelialen Zellkulturlinien aus Hoden infizierter Meerschweinchen. Hermann Mooser (1891-1971) erbrachte sodann den ersten mikroskopischen Erregernachweis im Warmblüter, nämlich in der Tunica vaginalis des Meerschweinchens ${ }^{177}$. Herald Rea Cox (geb. 1907) ${ }^{178}$ bewerkstelligte schließlich als erster die Züchtung der verschiedenen Rickettsien im Dottersack des bebrüteten Hühnereis. Erst dadurch wurde die Impfstoff herstellung und die Schutzimpfung in größerem Maßstabe möglich, dank der das Fleckfieber im Zweiten Weltkrieg unvergleichlich wirksamer als im Ersten zu beherrschen war ${ }^{179,180}$.

Die endgültige ätiologische Abgrenzung einer weiteren Rickettsiose, des Fünftage-, Schützengraben- oder Wolhynischen Fiebers, ist wiederum ein Verdienst von Henrique da Rocha Lima ${ }^{181}$. Die genauere klinische und epidemiologische Charakterisierung dieser seit 1915 bekannten ${ }^{182-184}$, auch als Werner-His'sche Krankheit bezeichneten Kriegsseuche gelang dem Stabsarzt Prof. Heinrich Werner (1874-1946) ${ }^{185-188}$ und dem Generaloberarzt und beratenden Heeresinternisten Prof. Wilhelm His jun. (1863-1934) ${ }^{189,190}$. Werner war ein Schüler von Stanislaus von Prowazek ${ }^{191}$; er arbeitete 1906-1913 am Hamburger Tropeninstitut, war dann kurze Zeit Chef des deutschen Gesundheitsdienstes in Kamerun und praktizierte nach dem Krieg als Internist in Berlin-Steglitz ${ }^{11,76,192}$. His, der Sohn des Basler Anatomen Wilhelm His sen., hatte als junger Assistent in Leipzig das nach ihm benannte atrioventrikuläre Reizleitungsbündel im Myokard entdeckt. Er übernahm den klinischen Lehrstuhl in Basel und wurde Nachfolger Ernst von Leydens in Berlin ${ }^{193,194}$. Seine Kriegserinnerungen sind unter dem Titel «Die Front der Ärzte» erschienen ${ }^{195}$.

Daß das Fünftagefieber eine Rickettsiose sei, wurde erstmals von Hans Willi Töpfer vermutet ${ }^{196}$. Der Armeepathologe und Oberarzt d. R. Alexander Schmincke (1877-1953) ${ }^{197}$ schlug 1917 als erster die Erregerbenennung Rickettsia quintana vor $^{198}$. Rickettsia quintana zeichnet sich im Gegensatz zum Erreger des Fleckfiebers dadurch aus, daß sie sich im Mitteldarm verschiedener Läusearten - nicht nur in dem der Kleiderlaus wie Rickettsia prowazekii - extrazellulär, d.h. im Darminhalt, also nicht in den Epithelzellen, aufhält. Sie ist pathogen für Menschen und Affen, nicht jedoch für kleine Laboratoriumstiere ${ }^{199,200}$. Ferner ist R. quintana auch nicht pathogen für die Laus; d.h. diese geht nicht zugrunde wie am Fleckfieber, sondern scheidet den Erreger vielmehr lebenslang und unbeschadet aus.

Daß zur erfolgreichen Bekämpfung des Fleckfiebers sowie des Fünftagefiebers die Läusebekämpfung bei Truppe und Zivilbevölkerung Voraussetzung ist, konnte nicht überzeugender begründet werden als durch die Forschungsergebnisse von da Rocha Lima. Die rasch verbesserten Entlausungsverfahren haben noch wäh- 
rend des Weltkrieges zu einer wirkungsvollen Eindämmung der Rickettsiosen geführt. Bereits 1917 hat da Rocha Lima auch vorgeschlagen, aus getrockneten und homogenisierten Läusen einen Impfstoff herzustellen ${ }^{201,202}$, ein Verfahren, auf dem die lange Zeit angewandte Technik der Impfstoffbereitung beruhte ${ }^{203}$ und das namentlich von Rudolf Weigl (1883-1957) 204 vervollkommnet wurde. Wenn es heute auch längst überholt und durch die Impfstoffgewinnung aus der Dottersackkultur ${ }^{179,180}$ ersetzt ist, so hat es damals immerhin die recht fragwürdige passive Immunisierung mit Rekonvaleszentenblut ${ }^{205}$ völlig verdrängt. Die letztere Methode war alles andere als gefahrlos. Besonders wenn etwa Blut von nicht entfieberten Spendern verwendet und die Inaktivierung vergessen wurde, kam es zu katastrophalen Zwischenfällen wie beispielsweise zu dem, den H. Hamdi (1873-1936) ${ }^{206}$ geschildert hat ${ }^{207}$.

1928 kehrte da Rocha Lima in seine Heimat zurück. Er wurde Abteilungsleiter an dem neuen Instituto Biologico in São Paulo und übernahm die Gesamtleitung dieser bundesstaatlichen Anstalt im Jahre 1933. 1939 war er Vizepräsident des 3. internationalen Mikrobiologen-Kongresses in New York. Er gehörte den mikrobiologischen, parasitologischen, pathologischen, tropenmedizinischen und dermatologischen Fachgesellschaften Deutschlands, Brasiliens, Venezuelas ${ }^{208}$, der Sowjetunion und der Vereinigten Staaten an. Mit seinen umfassenden Verdiensten war da Rocha Lima ein würdiger Empfänger des Ehrenzeichens des deutschen Roten Kreuzes und der Bernhard Nocht-Medaille (1921). Papst Pius XI. verlieh ihm 1925 die päpstliche Verdienstmedaille, und die deutsche Reichsregierung ernannte ihn 1938 zum Ritter des Roten Adlers. Zu seinen Ehren wurde 1947 die nomenklatorische Bezeichnung «Rochalimea» von A. Macchiavello für das dem Erreger des Fünftagefiebers vorbehaltene Rickettsien-Genus vorgeschlagen $^{209}$. Für die Abtrennung und Umbenennung von Rickettsia quintana in Rochalimea quintana plädierte auch A. Krieg ${ }^{210}$, nicht zuletzt deshalb, weil dieser Organismus - gleich anderen in Arthropoden lebenden Symbionten in künstlichen, unbelebten Medien züchtbar ist ${ }^{211}$.

1949 trat da Rocha Lima in den Ruhestand und verstarb, ohne eine noch geplante Europareise antreten zu können ${ }^{212}$, am 12. April 195628, 213,214.

\section{Literatur}

1 de Cerqueira Falcao, E., Henrique da Rocha Lima e a descoberta da Rickettsia prowazeki. Rev. Inst. Med. Trop. São Paulo 8 (1966), S.55-59.

2 de Cerqueira Falcao, E., A vida cientifica de Henrique da Rocha Lima. Rev. Brasil. Malariol. 19 (1968), S.353-358. 
${ }^{3}$ da Rocha-Lima, H.: Oswaldo G. Cruz. Arch. Schiffs- und Tropenhyg. 21 (1917), S.233237.

${ }^{4}$ Doty, R.B., Microbiology on stamps: The Cruz Institute. ASM News (Amer. Soc. Microbiol.) 41 (1975), S.720-722.

${ }^{5}$ Goldschmidt., R.B., Erlebnisse und Begegnungen. P.Parey: Hamburg-Berlin 1959.

${ }^{6}$ Hartmann, M.: S. von Prowazek. Arch. Protistenk. 36 (1915), S. I-XIX.

${ }^{7}$ Hatschek, B., Nachruf für Stanislaus v. Prowazek. Verh. zool. bot. Ges. Wien 65 (1915), S. 65-70.

${ }^{8}$ Jollos, V.: Stanislaus v. Prowazek. Biol. Zbl.35 (1915), S.337-341.

${ }^{9}$ Lipschütz, B.: S. v. Prowazek. Wien. klin. Wschr. 28 (1915), S.902-903.

10 Mayer, M., Professor S. v. Prowazek. Arch. Schiffs- und Tropenhyg. 19 (1915), S. 157-159.

${ }^{11}$ Olpp, G., Hervorragende Tropenärzte in Wort und Bild. O. Gmelin: München 1932.

${ }^{12}$ Samsinak, K., 1965 - jubilejni rok Stanislava Prowazka. Zpravy Ceskoslov. Spol. Parasitol.6 (1965), S.28-30.

${ }^{13}$ Kruta, V., Dictionary of scientific biography (C.C.Gillispie ed.), vol. XI, S.174-175. Ch. Scribner's Sons: New York 1975.

${ }^{14}$ Halberstaedter, L./S.v.Prowazek, Zur Ätiologie des Trachoms. Dtsch. med. Wschr.33 (1907), S.1285-1287.

${ }^{15}$ Halberstaedter, L./S.v.Prowazek, Zur Ätiologie des Trachoms. Berl. klin. Wschr. 46 (1909), S.1110-1113.

16 Prowazek, S./L.Halberstaedter, Chlamydozoa I. Zusammenfassende Übersicht. Arch. Protistenk.10 (1907), S.336-358.

17 v. Prowazek, S., Bemerkungen zur Kenntnis der pathogenen Mikroorganismen « Chlamydozoa ». Münch. med. Wschr.55 (1908), S.1016.

${ }^{18}$ Dyk, V./W.Eichler, Zum Gedenken an Stanislav Prowazek anläßlich seines 100. Geburtstags. Angew. Parasitol.19 (1978), S.230-232.

${ }^{19}$ Schadewaldt, H., Historischer Rückblick auf die Tropenmedizin in Deutschland. Ernst Rodenwaldt-Arch.2 (1975), S.135-155.

${ }^{20}$ Munk, F., Das medizinische Berlin um die Jahrhundertwende, hg. v. K. Munk. Urban \& Schwarzenberg: München-Berlin 1956.

${ }^{21}$ Rosenstein, P.: James Israel. Med. Klin.22 (1926), S.635-637.

22 da Rocha-Lima, H., Zur pathologischen Anatomie des Gelbfiebers. Verh. dtsch. pathol. Ges.15 (1912), S.163-182.

${ }^{23}$ da Rocha-Lima H., Zur pathologisch-anatomischen Diagnose des Gelbfiebers. Arch. Schiffs- und Tropenhyg.16 (1912), Beiheft 1, S.192-199.

${ }^{24}$ Couto, M./H. da Rocha-Lima, Gelbfieber. Menses Handbuch der Tropenkrankheiten, 3. Aufl., Bd.5, S.729-808. J.A. Barth: Leipzig 1929.

${ }^{25}$ da Rocha-Lima, H., Rückblickende Betrachtungen über die Entwicklung der Histodiagnose des Gelbfiebers. Festschrift Bernhard Nocht. J. J. Augustin: Glückstadt-Hamburg-New York 1937.

${ }^{26}$ Carter, H.R. (zit. n. Chiari, H.), Über Leberveränderungen bei Gelbfieber. Beitr. pathol. Anat. (Ziegler-Aschoff) 73 (1925), S.377-385.

${ }^{27}$ Torres, C.M., Sobre a importancia no diagnostico post mortem da febre amarella das lesoes microscopicas descriptas por H. da Rocha-Lima e por Hoffmann. Mem. Inst. Osw. Cruz 19 (1926), S.1-13. 
28 Anon.: Traços biograficos do Professor Rocha Lima. Arquiv. Inst. Biol. Sao Paulo 11 (1940), S. XVII-XXVI.

${ }^{29}$ da Rocha-Lima, H., Zur Histologie der Verruga peruviana. Verh. dtsch. pathol. Ges. 16 (1913), S.409-416.

${ }^{30}$ Mayer, M./H. da Rocha-Lima/H. Werner, Untersuchungen über Verruga peruviana. Münch. med. Wschr.60 (1913), S.739-741.

${ }^{31}$ da Rocha-Lima, H., Verruga peruviana. Oroyafieber. Menses Handbuch der Tropenkrankheiten, 3. Aufl., Bd.4, S.355-396. J. A. Barth: Leipzig 1926.

${ }^{32}$ da Rocha-Lima, H., Verruga peruviana oder Carrion'sche Krankheit (Oroyafieber). Handbuch der Haut- und Geschlechtskrankheiten (Jadassohn), Bd.12, Teil I, S.215-242 (1932).

33 da Rocha-Lima, H., Über das Verhalten des Erregers der brasilianischen Trypanosomiasis des Menschen in den Geweben. Verh. dtsch. pathol. Ges. 15 (1912), S. 454-459.

${ }^{34}$ Mayer, M./H. da Rocha-Lima, Zur Entwicklung von Schizotrypanum cruzi in Säugetieren. Arch. Schiffs- und Tropenhyg. 16 (1912), Beiheft 4, S.376(90)-380(94).

${ }^{35}$ Chagas, C./E. Villela/H. da Rocha-Lima, Amerikanische Trypanosomenkrankheit. Chagas-Krankheit. Menses Handbuch der Tropenkrankheiten, 3.Aufl., Bd.5, S.673-728. J.A.Barth: Leipzig 1929.

${ }^{36}$ da Rocha-Lima, H., Exotische Blastomykosen. Handbuch der Haut- und Geschlechtskrankheiten (Jadassohn), Bd.12, Teil I, S.366-398 (1932).

37 da Rocha-Lima, H., Über Blastomykose, venerisches Granulom und klimatische Bubonen. Arch. Dermatol. Syphilol. 145 (1924), S.312-317.

${ }^{38}$ Mayer, M./H. da Rocha-Lima, Protozoen und Haut. Handbuch der Haut- und Geschlechtskrankheiten (Jadassohn), Bd.2, S.1-20 (1932).

${ }^{39}$ Darling, S.T., A protozoan general infection producing pseudotubercles in the lungs and focal necroses in the liver, spleen and lymphnodes. J.Amer. Med. Ass.46 (1906), S. 1283-1285.

${ }^{40}$ da Rocha-Lima, H., Histoplasmosis und epizootische Lymphangitis. Arch. Schiffs- und Tropenhyg.16 (1912), Beiheft 1, S.79-85.

41 da Rocha-Lima, H., Beitrag zur Kenntnis der Blastomykosen; Lymphangitis epizootica und Histoplasmosis. Zbl. Bakt., I. Abt. Orig. 67 (1913), S. 233-249.

42 da Rocha-Lima, H./J.Reis/K. Silberschmidt, Methoden der Virusforschung. Handb. biol. Arbeitsmethoden (E. Abderhalden), Abt. XII, Teil 2, S.1135-1489. Urban \& Schwarzenberg: Berlin-Wien 1939.

43 da Rocha-Lima, H., Zur Ätiologie des Fleckfiebers. Z. allg. Pathol. 27 (1916), S.45-50.

${ }^{44}$ da Rocha-Lima, H., Untersuchungen über Fleckfieber. Münch. med. Wschr.63 (1916), S. 1381-1384.

45 da Rocha-Lima, H., Die Übertragung des Rückfallfiebers und des Fleckfiebers. Dtsch. med. Wschr. 45 (1919), S.732-734.

46 da Rocha-Lima, H./H.Sikora, Methoden zur Untersuchung von Läusen als Infektionsträger. Handb. biol. Arbeitsmeth. (E.Abderhalden), Abt. XII, Teil 1, S.769-814. Urban \& Schwarzenberg: Berlin-Wien 1925.

47 Wilson, G.S./A.A.Miles, Topley and Wilson's principles of bacteriology, virology and immunology, 6th ed. Edw. Arnold: London 1975.

${ }^{48}$ Anon.: Howard Taylor Ricketts. J. Amer. Med. Ass. 54 (1910), S. 1640. 
49 Anon.: An act of homage to a medical martyr. Lancet I/1911, S. 520.

50 Asimov, I., Biographische Enzyklopädie der Naturwissenschaften und derTechnik. Herder: Freiburg 1973.

${ }^{51}$ Mullen, P. C., Dictionary of scientific biography (C. C. Gillispie ed.). Ch. Scribner's Sons: New York 1975.

${ }^{52}$ New York Times, 18.Dez.1959, S.29.

${ }^{53}$ Deaths: Wilder, Russell Morse. J. Amer. Med. Ass. 172 (1960), S. 1307.

54 Nécrologue: Charles Nicolle. Arch. Inst. Past. Tunis 25 (1936), S. 201-248.

${ }^{55}$ Nicolle, Ch.: Ernest Conseil (1879-1930). Arch. Inst. Past. Tunis 19 (1930), S.341-375.

${ }_{56}$ Nicolle, Ch.: A. Conor. Arch. Inst. Past. Tunis 9 (1914), o. S.

${ }^{57}$ Ricketts, H.T., The transmission of Rocky Mountain spotted fever by the bite of the wood tick (Dermacentor occidentalis). J. Amer. Med. Ass. 47 (1906), S. 358.

${ }^{58}$ Ricketts, H.T./R.M. Wilder, The relation of typhus fever (tabardillo) to Rocky Mountain spotted fever. Arch. int. Med.5 (1910), S.361-370.

${ }^{59}$ Conor, A./A. Bruch, La fièvre boutonneuse de Tunisie. Arch. Inst. Past. Tunis 5 (1910), S.126-133.

${ }^{60}$ Conor, A./A. Bruch, Une fièvre éruptive observée en Tunisie. Bull. Soc. Path. exot. 3 (1910), S.492-496.

${ }^{61}$ Nicolle, Ch./Ch. Comte/E. Conseil, Transmission expérimentale du typhus exanthématique par le pou du corps. C. r. Acad. Sci. Paris 149 (1909), S.486-489.

${ }^{62}$ Nicolle, Ch., Réproduction expérimentale du typhus exanthématique chez le singe. C. r. Acad. Sci. Paris 149 (1909), S.157-160.

${ }^{63}$ Nicolle, Ch./E. Conseil/A. Conor, Le typhus expérimental du cobaye. C. r. Acad. Sci. Paris 152 (1911), S.1632-1634.

${ }^{64}$ Dach, E.F., Selbstversuche von Ärzten mit lebenden Krankheitserregern. CIBA Zeitschrift Nr.5, 1934.

${ }^{65}$ Doerr, R./R.Pick, Experimentelle Untersuchungen über Infektion und Immunität bei Fleckfieber. Wien. klin. Wschr.31 (1918), S. 829-834.

${ }^{66}$ Zinsser, H., Rats, Lice, and History. Little, Brown \& Co.: Boston 1935.

67 Mooser, H., Das epidemische Fleckfieber, S. 851-866 in: Virus- und Rickettsieninfektionen des Menschen (R.Haas \& O.Vivell ed.). J.F.Lehmann: München 1965.

${ }^{68}$ Hegler, C./S.v. Prowazek, Untersuchungen über Fleckfieber. Vorl. Bericht. Berl. klin. Wschr.50 (1913), S. 2035-2040.

${ }^{69}$ Prowazek, S. von/C. Hegler, Über Flecktyphus. Beitr. Klin. Infektionskr.4 (1915), S. 5-31.

${ }^{70}$ Budelmann, D.: Prof. Dr. med. Carl Hegler. Med. Klin.40 (1944), S. 149.

${ }^{71}$ Bennhold, H.: Carl Hegler. Dtsch. med. Wschr.70 (1944), S.163.

72 Domagk, G./C. Hegler, Chemotherapie bakterieller Infektionen, 3. Aufl. S. Hirzel: Leipzig $1941 / 1944$.

${ }^{73}$ Hegler, C., Praktikum der wichtigsten Infektionskrankheiten, 4. Aufl. G.Thieme: Wiesbaden 1946.

${ }^{74}$ Mühlens, P., Vier Jahre Kriegshygiene in der Türkei und auf dem Balkan, S.141-160 in: Vor 20 Jahren, 2. Folge (Dtsch. med. Wschr. ed). G. Thieme: Stuttgart 1935.

${ }^{75}$ Wolter, F., Über das Auftreten von Flecktyphusepidemien in Truppen- und Gefangenenlagern. Berl. klin. Wschr.52 (1915), S.1045-1048. 
${ }^{76}$ Fischer I., Biographisches Lexikon der Ärzte der letzten fünfzig Jahre, 2 Bde. Urban \& Schwarzenberg: Berlin-Wien 1932/33.

77 Kürschners Deutscher Gelehrtenkalender. W. de Gruyter: Berlin-Leipzig 1931.

${ }^{78}$ Kürschners Deutscher Gelehrtenkalender. W. de Gruyter: Berlin 1961.

${ }^{79}$ Jürgens, G., Zur Epidemologie des Fleckfiebers. Berl. klin. Wschr. 52 (1915), S. 654-661.

${ }^{80}$ Jürgens, G. J., Infektionskrankheiten. 1920.

81 Jürgens, G., Grundlagen der Epidemiologie. J.A. Barth: Leipzig 1936.

82 Jürgens, G. J., Das Fleckfieber. Berlin 1916.

${ }^{83}$ Otto, Beobachtungen bei einer Fleckfieberepidemie. Dtsch. med. Wschr.41 (1915) S. 1325-1328, 1357-1359.

${ }^{84}$ Skutetzky, A., Die Flecktyphusepidemie im k.u.k. Kriegsgefangenenlager in Marchtrenk, O. Österr., im Jahre 1915. Wien. klin. Wschr. 28 (1915), S. 887-891.

${ }^{85}$ Rondke, Die Fleckfieberepidemie im Görlitzer Kriegsgefangenenlazarett. Med. Klin.11 (1915), S.1152-1155.

${ }^{86}$ Sanitätsbericht über das Deutsche Heer im Weltkriege 1914/1918. E.S. Mittler \& Sohn: Berlin 1935.

${ }^{87}$ Flügge, C., Schutzkleidung gegen Flecktyphusübertragung. Med. Klin.52 (1915), S. $420-421$.

${ }^{88}$ Wasielewski, Th. v., Über die Vorbeugung von Fleckfieberübertragungen auf Ärzte und Pfleger. Münch. med. Wschr.62 (1915), S.627-628.

${ }^{89}$ Schilling, V., Kriegshygienische Erfahrungen in der Türkei. Arch. Schiffs- und Tropenhyg. 25 (1921), Beiheft 3, S.5-41.

${ }^{90}$ Fejgin, B., Sur la persistance du virus du typhus exanthématique dans les poux. C. r. Soc. Biol.123 (1936), S.37-39.

${ }^{91}$ Starzyk, J., Vitalité, toxicité et pouvoir d'immunisation de Rickettsia prowazeki conservées hors de l'organisme du pou, en milieu liquide et en milieu sec. C. r. Soc. Biol.123 (1936), S.1221-1225.

92 Weyer, F., Über die Lebensdauer von Rickettsien im Kot der Laus. Arch. Inst. Past. Tunis 36 (1959), S.411-428.

${ }^{93}$ Neue Deutsche Biographie (hrsg. Histor. Kommission d. bayr. Akad. d. Wiss.). Duncker \& Humblot: Berlin $1953 \mathrm{ff}$.

${ }^{94}$ Klemperer, G./W.Zinn, Zur Diagnose und Prophylaxe des Fleckfiebers. Therap. Gegenw. 56 (1915), S.41-45.

95 Wolter, F., Über den Flecktyphus als Kriegsseuche. Berl. klin. Wschr. 52 (1915), S. 807$809,851-854$.

${ }^{96}$ Jochmann, G., Lehrbuch der Infektionskrankheiten für Ärzte und Studierende. J.Springer : Berlin 1914.

97 Jochmann, G./C. Hegler, Lehrbuch der Infektionskrankheiten, 2. Aufl. J.Springer: Berlin 1924.

${ }^{98}$ Kauffmann, F., Zur Geschichte der Salmonella-Forschung. Zbl. Bakt., I. Abt. Orig. 201 (1966), S. 44-48.

${ }^{99}$ Sackmann, W.: Reiner Müller, 20. Mai 1879 - 5. Juli 1953. Dtsch. med. Wschr.104 (1979), S. 747-748.

100 Neukirch, P., Über Paratyphusbakterien im Blute bei ruhrähnlichen Erkrankungen in der Türkei. Berl. klin. Wschr.54 (1917), S.360-364. 
101 Neukirch, P., Über menschliche Erkrankungen durch Bazillen der Gläßer-Voldagsengruppe in der Türkei. Z. Hyg.85 (1918), S. 103-145.

${ }^{102}$ His, W., Medizinisches aus der Türkei. Berl. klin. Wschr.55 (1918), S.964-967.

103 Neukirch, P., Über Versuche prophylaktischer Impfung gegen Fleckfieber. Med. Klin. 13 (1917), S.300-301.

${ }^{104}$ Huntemüller, Als beratender Hygieniker in der asiatischen Türkei. Med. Klin. 15 (1919), S.1101-1104, 1129-1131, 1158-1160, 1186-1187, 1215-1216, 1240-1241.

105 Personalia, Dtsch. med. Wschr. 47 (1921), S. 1596.

106 Personalia, Münch. med. Wschr. 69 (1922), S. 68.

${ }^{107}$ Brugsch, Th.: Arthur Pappenheim. Fol. haematol.21 (1917), S.79-90.

108 v.d.Goltz, C., Denkwürdigkeiten, 2. Aufl. E. \& S. Mittler: Berlin 1932.

${ }^{109}$ Goldberger, J./J.F. Anderson, The transmission of typhus fever, with special reference to transmission by the head louse (Pediculus capitis). Publ. Hlth. Repts. 27 (1912), S. 297-307.

110 Ricketts, H.T./R.M. Wilder, The transmission of the typhus fever of Mexico (Tabardillo) by mean of the louse. J. Amer. Med. As.54 (1910), S.1304-1307.

111 Ricketts, H.T./R.M. Wilder, The etiology of the typhus fever (Tabardillo) of Mexico. J. Amer. Med. Ass. 54 (1910), S.1373-1375.

112 Nicolle, Ch., Recherches expérimentales sur le typhus exanthématique, entreprises à l'Institut Pasteur de Tunis pendant l'année 1909. Ann. Inst. Past.24 (1910), S. 243-275.

113 Nicolle, Ch., Recherches expérimentales sur le typhus exanthématique, entreprises à l'Institut Pasteur de Tunis pendant l'année 1910. Ann. Inst. Past. 25 (1911), S.97-144.

${ }^{114}$ Nicolle, Ch./E. Conseil/A. Conor, Recherches expérimentales sur le typhus exanthématique, entreprises à l'Institut Pasteur de Tunis pendant l'année 1911. Ann. Inst. Past. 26 (1912), S.250-280, 332-350.

115 da Rocha-Lima, H., Beobachtungen bei Flecktyphusläusen. Arch. Schiffs- und Tropenhyg. 20 (1916), S.17-31.

116 da Rocha-Lima, H., Die Ursache des Fleckfiebers. Umschau 20 (1916), S.1005-1010.

117 Sikora, H., Beiträge zur Anatomie, Physiologie und Biologie der Kleiderlaus. Arch. Schiffs- und Tropenhyg. 20 (1916), Beiheft 1, S.1-76.

118 Anon.: Angew. Parasitol.11 (1970), S. 63.

119 Anon.: Todesfälle. Angew. Parasitol.19 (1978), S. 64.

${ }^{120}$ Girard, G.: Henry Foley (1871-1956). Bull. Soc. Path. exot.50 (1957), S.3-5.

${ }^{121}$ Sergent, E./H.Foley/C. Vialatte, Sur des formes microbiennes abondantes dans le corps de poux infectés par le typhus exanthématique, et toujours absentes dans les poux témoins, non typhiques. C. r. Soc. Biol. 66/II (1914), S.101-103.

${ }^{122}$ Geigy, R./A.Herbig, Erreger und Überträger tropischer Krankheiten, S.326-334. Verlag f. Recht \& Gesellschaft: Basel 1955.

${ }^{123}$ da Rocha-Lima, H., Zur Ätiologie des Fleckfiebers. Vorläufige Mitteilung. Berl. klin. Wschr.53 (1916), S. 567-569.

124 da Rocha-Lima, H., Über die Ätiologie des Fleckfiebers. Berlin. Klin. 29 (1919), S.1-26.

125 da Rocha-Lima, H., Die Ätiologie des Fleckfiebers. Erg. allg. Pathol.19 (1919), 1.Abt., S. 159-306.

${ }^{126}$ Mayer, M., Die Ergebnisse der experimentellen Flecktyphusforschung. Naturwiss.4 (1916), S.1005-1010. 
127 da Rocha-Lima, H., Zur Ätiologie des Fleckfiebers. Dtsch. med. Wschr.42 (1916), S. 1353-1354.

${ }^{128}$ Morton, L.T., A medical bibliography (Garrison and Morton) $3 \mathrm{~d}$ ed. A. Deutsch: London 1970.

${ }^{129}$ Töpfer, H./H.Schüßler, Zur Ätiologie des Fleckfiebers. Dtsch. med. Wschr.42 (1916), S.1157-1158.

130 Töpfer, H., Der Fleckfiebererreger in der Laus. Dtsch. med. Wschr.42 (1916), S.12511254.

131 da Rocha-Lima, H., Zur Ätiologie des Fleckfiebers. Dtsch. med. Wschr. 43 (1917), S. 50.

132 Töpfer, H., Zur Ätiologie des Fleckfiebers. Dtsch. med. Wschr.43 (1917), S. 50.

133 da Rocha-Lima, H., Untersuchungen über Fleckfieber. Münch.'med. Wschr.63 (1916), S.1381-1384.

${ }^{134}$ Spät, W., Zur Frage des Flecktyphus auf dem galizischen Kriegsschauplatz. Wien. klin. Wschr.28 (1915), S.1103-1106.

135 Weil, E./W.Spät, Die Bedeutung der Widalschen Reaktion für die Diagnose des Flecktyphus. Wien. klin. Wschr.28 (1915), S.207-208.

${ }^{136}$ Bail, O.: Edmund Weil. Z. Immunforsch. 35 (1923), S.2-24.

137 Craigie, J.: Arthur Felix, 1887-1956. Biogr. Mem. Fellows Roy. Soc.3 (1957), S.53-79.

138 Wilson, G.S.: Arthur Felix, 3 rd April 1887 - 14th January 1956. J. Path. Bact. 73 (1957), S. 281-295.

139 Klieneberger-Nobel, E., Pionierleistungen für die medizinische Mikrobiologie. G. Fischer: Stuttgart-New York 1977.

140 Weil, E./A.Felix, Zur serologischen Diagnose des Fleckfiebers. Wien. klin. Wschr.29 (1916), S.33-35.

${ }^{141}$ Felix, A., Die Serodiagnostik des Fleckfiebers. Wien. klin. Wschr.29 (1916), S.974-978.

${ }_{142}$ Poggendorff, J.C., Biographisch-literarisches Handwörterbuch der exakten Naturwissenschaften, VIIa. Akademie-Verlag: Berlin 1955.

${ }^{143}$ Hamburger R./R.Bauch, Untersuchungen über die Weil-Felixsche Reaktion. Dtsch. med. Wschr.43 (1917), S.1130-1131, 1227-1229.

144 Bail, O., Nachsatz zur Korrektur von (140). Wien. klin. Wschr.29 (1916), S. 35.

${ }^{145}$ Felix, A., Serologische Untersuchungen an Fleckfieberkranken aus der asiatischen Türkei. Z. Immunforsch.26 (1917), S.602-619.

146 White, P.B., The O-receptor complex of B. proteus $\mathrm{OX}_{19}$. Brit. J. exp. Pathol.14. (1933), S. 145-148.

147 Felix, A./M. Rhodes, Serological varieties of typhus fever. J. Hyg. Camb.31 (1931), S. 225-246.

${ }^{148}$ Castaneda, M.R./S.Zia, The antigenic relationship between Proteus X-19 and Typhus Rickettsiae. J. exp. Med.58 (1933), S.55-62.

${ }^{149}$ Castaneda, M.R., The antigenic relationship between Proteus X-19 and typhus rickettsia. II. A study of the common antigenic factor. J. exp. Med.60 (1934), S.119-125.

150 Sackmann, W.: Viktor Karl Ruß, 22.Feb.1879 - 4. Mai 1956. Österr. Ärztezeitg.34 (1979), S.152-156.

${ }^{151}$ Ruß, V.K./L. Kirschner, Studien über das Fleckfiebervirus. Z. Hyg.92 (1921), S. 38-50.

152 Obituary: William James Wilson. Lancet I/1954, S.1036-1037.

153 Obituary: W. J. Wilson. Brit. med. J. I/1954, S.1329. 
${ }^{154}$ Wilson, W.J./E.McV.Blair, Combination of bismuth and sodium sulfite affording enrichment and selective medium for typhoid-paratyphoid groups of bacteria. J. Path. Bact.29 (1926), S.310-311.

${ }^{155}$ Wilson, W. J., On heterologous agglutinins, more particularly those present in the blood serum of cerebrospinal fever and thyphus fever cases. J. Hyg. Camb. 9 (1909), S. 316-340.

${ }^{156}$ Wilson, W. J., The Wilson-Weil-Felix reaction in typhus fever. J. Hyg. Camb. 19 (1920), S. 115-130.

${ }^{157}$ Weil, E./A.Felix, Über die Doppelnatur der Receptoren beim Paratyphus B. Wien. klin. Wschr.31 (1918), S.986-988.

${ }^{158}$ Weil, E./A.Felix, Über den Doppeltypus der Rezeptoren in der Typhus-ParatyphusGruppe. Z. Immunforsch.29 (1920), S.24-91.

${ }^{159}$ Smith, Th./A.L. Reagh, The non-identity of agglutinins acting upon the flagella and upon the body of bacteria. J. med. Res. Boston 10 (1903), S. 89 (zit. 167).

${ }^{160}$ Beyer, H. G./A.L. Reagh, The further differentiation of flagellar and somatic agglutinins. J. med. Res. Boston 12 (1904), S.313 (zit. 167).

${ }^{161}$ Joos, A., Untersuchungen über die verschiedenen Agglutinine des Typhusserums. Zbl. Bakt., I. Abt. Orig. 33 (1903), S.762-783.

${ }^{162}$ Felix, A./R.M.Pitt, A new antigen of B. typhosus. Its relation to virulence and to active and passive immunisation. Lancet II/1934, S.186-191.

${ }^{163}$ Weil, E., Paratyphus B-ähnliche Krankheitserreger (Typus Suipestifer-Voldagsen) in Albanien. Wien. klin. Wschr.30 (1917), S.1061-1063.

${ }^{164}$ Bergey's Manual of Determinative Bacteriology (R.E.Buchanan \& N.E.Gibbons ed.), 8 th ed., S.300. Williams \& Wilkins Co.: Baltimore 1974.

${ }^{165}$ Hirschfeld, L., A new germ of Paratyphoid. Lancet I/1919, S.296-297.

${ }^{166}$ Breinl, F./E. Chrobok, Die Erreger des Fleckfiebers und des Felsengebirgsfiebers. Zbl. Bakt., I. Abt. Orig.138 (1937), S.129-137.

167 Kauffmann, F., Das Fundament zur Geschichte und Bedeutung der Salmonella- und Escherichia-Forschung. E. Munksgaard: Kopenhagen 1978.

${ }^{168}$ Cannon, W.B., Foreword. Arch. Pathol.30 (1940), S.1-6.

169 Janeway, Ch. A.: S.Burt Wolbach 1880-1954. Trans. Assoc. Amer. Phys. 67 (1954), S. 30-35.

170 Warren, Sh.: Simeon Burt Wolbach, 3 rd July 1880 - 19 th March 1954. J. Path. Bact. 68 (1954), S.656-657.

171 Farber, S./Ch. L.Maddock: S. Burt Wolbach M.D., 1880-1954. Arch. Pathol.59 (1955), S. 624-630.

172 Deaths: Schlesinger, Monroe Jacob. J. Amer. Med. Ass. 157 (1955), S. 1036.

173 J.B. A.: Francis Winslow Palfrey 1876-1953. New. Engld. J. Med. 249 (1953), S. 383-384.

174 Obituary: Dr. John L.Todd. Canad. Med. Ass. J.61 (1949), S. 544.

175 Wolbach, S.B./J.L.Todd/F. W.Palfrey, The Etiology and Pathology of Typhus. Report of the typhus research commission of the League of the Red Cross Societies to Poland. Harvard Univ. Press: Cambridge Mass. 1922.

176 Wolbach, S.B./M.J.Schlesinger, The cultivation of the micro-organisms of Rocky Mountain Spotted Fever (Dermacentroxenus rickettsi) and of Typhus (Rickettsia prowazeki) in tissue plasma cultures. J. med. Res. Boston 44 (1923), S.231-256. 
177 Mooser, H., Ein Beitrag zur Ätiologie des mexikanischen Fleckfiebers. Arch. Schiffs- und Tropenhyg. 32 (1928), S.261-264.

${ }^{178}$ Current Biography Yearbook 1961 (Ch.Moritz ed.). H.W.Wilson Co.: New York, S.118-120.

${ }^{179}$ Cox., H.R., Use of yolk sac of developing chick embryo as medium for growing Rickettsiae of Rocky Mountain Spotted Fever and Typhus groups. Publ. Hlth. Repts. Wash. 23 (1938), S.2241-2247.

180 Cox, H.R., Cultivation of Rickettsiae of the Rocky Mountain Spotted Fever, Typhus and Q Fever groups in the embryonic tissues of developing chicks. Science 94 (1941), S. 399-403.

181 Munk F./H. da Rocha-Lima, Klinik und Ätiologie des sogen. «Wolhynischen Fiebers» (Werner-His'sche Krankheit). Münch. med. Wschr.64 (1917), S. 1422-1426.

${ }^{182}$ Graham, J.H.P., A note on a relapsing febrile illness of unknown origin. Lancet II/1915, S. 703-704.

${ }^{183}$ Hunt, G.H./A. C. Rankin, Intermittent fever of obscure origin, occurring among British soldiers in France. The so-called "trench fever". Lancet II/1915, S.1133-1136.

${ }^{184}$ McNee, J.W./A.Renshaw/E.H.Brunt, "Trench Fever": A relapsing fever with the British forces in France. Brit. med. J. I/1916, S.225-234.

${ }^{185}$ Werner, H., Über rekurrierendes Fieber (Rekurrens ?) mit Fünftageturnus, Fünftagefieber, aus dem Osten (Vorl. Mitt.). Münch. med. Wschr.63 (1916), S. 402.

186 Werner, H./E. Haenßler, Über Fünftagefieber, febris quintana. Münch. med. Wschr.63 (1916), S.1020-1021.

187 Werner, H./F. Benzler/O. Wiese, Zur Ätiologie des Fünftagefiebers. Münch. med. Wschr. 63 (1916), S.1369-1370.

188 Werner, H., Zur Diagnose des Fünftagefiebers. Dtsch. med. Wschr.68 (1942), S.934-935.

${ }^{189}$ His, W., Über eine neue, periodische Fiebererkrankung (Febris Wolhynica). Berl. klin. Wschr.53 (1916), S. 738-739.

${ }^{190} \mathrm{His}, \mathrm{W}$. , Referierabend der deutschen und österreichisch-ungarischen Militärärzte in Jerusalem. Wien. klin. Wschr.30 (1917), S.995-996.

191 v. Prowazek, S./H. Werner, Zur Kenntnis der sog. Flagellaten. Arch. Schiffs- und Tropenhyg. 18 (1914), Beiheft 5, S.155-170 (311-326).

192 Kleine, F.K., Prof. Heinrich Werner zum 70. Geburtstag. Dtsch. med. Wschr.70 (1944), S. 253.

${ }^{193}$ Staehelin, R.: Wilhelm His, der Kliniker. Schweiz. med. Jahrb.8 (1936), S. XXIIIXXXIV.

194 Staub, H.: Wilhelm His jun. 1863-1934, S.284 in: Professoren der Universität Basel aus fünf Jahrhunderten (hrsg. von A. Staehelin). Fr. Reinhardt A. G.: Basel 1960.

195 His, W., Die Front der Ärzte. Velhagen \& Klasing: Bielefeld-Leipzig 1931.

196 Toepfer, H., Zur Ursache und Übertragung des Wolhynischen Fiebers. Münch. med. Wschr.63 (1916), S.1495-1496.

197 Doerr, W., Alexander Schmincke zum Gedächtnis (1877-1953). Zbl. allg. Path. pathol. Anat.91 (1954), Abt. Lit., S.1-9.

${ }^{198}$ Schmincke, A., Histopathologischer Befund in Roseolen der Haut bei Wolhynischem Fieber. Münch. med. Wschr.64. (1917), S.961. 
199 da Rocha-Lima, H., Klinik und Ätiologie des sogenannten «Wolhynischen Fiebers» (Werner-His'sche Krankheit). II. Ergebnis der ätiologischen Untersuchungen und deren Beziehungen zur Fleckfieberforschung. Med. Klin.13 (1917), S.1227.

200 Mooser, H., Rickettsiosen, S.1204-1239 in: Die Infektionskrankheiten des Menschen und ihre Erreger (hrsg. von A.Grumbach und W. Kikuth). G. Thieme: Stuttgart 1958.

201 da Rocha-Lima, H., Die Schutzimpfung gegen das Fleckfieber. Med. Klin.13 (1917), S. 1147-1150.

202 da Rocha-Lima, H., Schutzimpfungsversuche gegen Fleckfieber. Münch. med. Wschr. 65 (1918), S. 1454-1456.

${ }^{203}$ Weigl., R., Die Methoden der aktiven Fleckfieberimmunisierung. Bull. Int. Acad. Polon. sci. et lettres (cl. Méd.) (1930), S.25-62.

204 Eyer, H., In memoriam Rudolf Weigl (1883-1957). Zbl. Bakt., I. Abt. Orig.171 (1958), S. 377-379.

${ }^{205}$ Mayer, G., Welches ist die beste Fleckfieberbehandlung? Z. ärztl. Fortbild.28 (1931), S. 430-431.

${ }^{206}$ Sevki Aygün, K.: H.Hamdi (1873 - 13.III.1936). Verh. dtsch. pathol. Ges.29 (1936), S. 367-369.

${ }^{207}$ Hamdi, H., Über die Ergebnisse der Immunisierungsversuche gegen Typhus exanthematicus. Z. Hyg. 82 (1916), S.235-242.

${ }^{208}$ Briceno Maaz, T., El Profesor Henrique da Rocha Lima ha muerto. Gaceta med. Caracas 64. (1957), S.439-44.1.

${ }^{209}$ Macchiavello, A., Notes on the taxonomy of the Rickettsias and the classification of the Rickettsioses. Prim. Reunion interameric. del Tifo, Mexico 1947, S.405-426.

${ }^{210}$ Krieg, A., Grundlagen der Insektenpathologie. D. Steinkopff: Darmstadt 1961.

${ }^{211}$ Vinson, J.W./H.S.Fuller, Studies on trench fever. I. Propagation of rickettsia-like microorganisms from a patient's blood. Path. Microbiol.24 (1961), Suppl., S. 152-166.

${ }^{212}$ Editorial: Da Rocha Lima zum Gedächtnis. Z. Tropenmed. Parasitol.7 (1956), Heft 2, o.S.

${ }^{213}$ Weyer, F., Zum Tode von Henrique da Rocha Lima. Desinf. Gesundheitswes. 48 (1956), S. 145-146.

${ }^{214}$ Weyer, F., Zur Entdeckungsgeschichte des Fleckfiebererregers. Z. Tropenmed. Parasitol. 17 (1966), S.478-483.

\section{Summary}

At the occasion of the one hundredth birthday of Henrique da Rocha Lima (1879-1956) the autor describes the history of typhusresearch, especially in Europe about the time of World War I.

Dr. med. vet. Werner Sackmann

Sieglinweg 10

CH-4125 Riehen 